\title{
LX-Shore : Un nouveau modèle d'évolution du trait de côte pour les littoraux sableux dominés par l'action des vagues
}

\section{Arthur ROBINET ${ }^{1}$, Déborah IDIER ${ }^{1}$, Bruno CASTELLE ${ }^{2}$, Vincent MARIEU $^{2}$}

1. Bureau de Recherches Géologiques et Minières,

3 avenue Claude Guillemin, BP 36009, 45060 Orléans Cedex 2, France.

a.robinet@brgm.fr ; d.idier@brgm.fr

2. CNRS, Université de Bordeaux, UMR 5805 EPOC,

Allée Geoffroy Saint-Hilaire, CS 50023, 33615 Pessac Cedex, France.

bruno.castelle@u-bordeaux.fr ; vincent.marieu@u-bordeaux.fr

\section{Résumé :}

Comprendre et prévoir les évolutions du trait de côte est crucial pour informer et guider les gestionnaires du littoral. Jusqu'à très récemment la plupart des modèles de trait de côte ne permettaient pas de répondre à ces objectifs le long des littoraux sableux dominés par l'action des vagues du fait de limitations numériques et/ou physiques. Cette étude présente un nouveau modèle numérique à complexité réduite, nommé LX-Shore, permettant de simuler les évolutions du trait de côte le long de ces littoraux, sur des échelles de temps allant de l'heure à quelques décennies, et avec des temps de calcul très réduits. Ce modèle est applicable à la plupart des géométries de côtes sableuses (ex. : flèches sableuses, îles) et prend en compte l'existence de zones non-érodables (ex. : ouvrages de défense, caps rocheux). LX-Shore s'appuie sur une approche one-line originale, où les évolutions du trait de côte résultent non seulement des gradients de transport sédimentaire longshore, mais aussi du transport sédimentaire cross-shore dû à la variabilité temporelle de l'énergie des vagues incidentes. LX-Shore est couplé avec le modèle spectral de vagues SWAN pour simuler la transformation des vagues au-dessus de bathymétries complexes et assurer une rétroaction de l'évolution du trait de côte sur la propagation des vagues. Les cas d'application présentés montrent par exemple que LX-Shore est capable de simuler la formation d'instabilités du trait de côte (ex. : flèches sableuses) ou résultant de l'implémentation de structures en dur (ex. : épis et caps rocheux) sur une large gamme d'échelles temporelles. Cet outil ouvre la voie vers : (1) une meilleure évaluation des processus physiques contrôlant les évolutions du trait de côte et de leurs contributions respectives, (2) et la réalisation de projections.

Mots-clés : Modèle numérique, Complexité réduite, Trait de côte, Littoraux sableux, Transport sédimentaire, Transport longshore, Modèle cross-shore d'équilibre, Vagues, SWAN. 


\section{Thème 2 - Dynamique sédimentaire}

\section{Introduction}

Dans le contexte actuel de changement climatique et d'augmentation de la pression démographique en zone littorale, il devient crucial de bien caractériser les processus physiques à l'origine de la dynamique des littoraux sableux et d'être capable de prédire les évolutions futures du trait de côte sur une large gamme d'échelles spatiotemporelles.

Au cours des dernières décennies, de nombreux modèles morphodynamiques basés sur une description fine des processus physiques ont été développés pour simuler et prédire à terme les évolutions morphologiques de la zone littorale, incluant les évolutions du trait de côte (LESSER et al., 2004 ; ROELVINK et al., 2009). Pour le cas des littoraux sableux dominés par l'action des vagues, ces modèles complexes échouent encore à simuler les évolutions du trait de côte sur le moyen (année/décennie) et long-terme (siècle) du fait de limitations physiques et numériques (cascade d'erreurs au travers des échelles et temps de calcul très longs). Pour ces échelles, l'utilisation de modèles numériques de trait de côte à complexité réduite représente une alternative pour simuler des évolutions de manière fiable et avec des temps de calcul raisonnables (VITOUSEK et al., 2017).

Dans le cas des littoraux sableux dominés par l'action des vagues, les évolutions du trait de côte sont généralement dominées sur le moyen et long-terme par les gradients longshore spatiaux de transport sédimentaire longshore, et sur le plus court-terme par les processus cross-shore liés à la variabilité temporelle des caractéristiques des vagues incidentes. Cependant, les contributions respectives des processus longshore et des processus cross-shore dans les évolutions du trait de côte varient aussi avec le climat de vagues et les caractéristiques géologiques du site et leur quantification reste parfois problématique (HARLEY et al., 2015). Dans le cas de plages ouvertes, les évolutions induites sur le court-terme par les processus cross-shore, avec une rapide érosion en période de tempête suivie d'une lente phase de reconstruction, cascadent parfois au travers des échelles temporelles et expliquent une part significative de la variabilité interannuelle et/ou de la tendance long-terme (ROBINET et al., 2016). La prise en compte des processus longshore et cross-shore dans les modèles de trait de côte devient une nécessité pour évaluer correctement les processus moteurs des évolutions du trait de côte et permettre les prédictions futures. Or, jusqu'à récemment, les processus longshore et cross-shore ont été traités séparément dans les modèles à complexité réduite.

Cette communication vise à présenter un nouveau modèle de trait de côte répondant à cette problématique et nommé LX-Shore (section 2). Plusieurs cas d'application sont présentés pour illustrer ses capacités (section 3). Une description plus exhaustive de LX-Shore est fournie dans ROBINET (2017) et ROBINET et al. (en révision). 


\section{XVèmes Journées Nationales Génie Côtier - Génie Civil \\ La Rochelle, 29 au 31 mai 2018}

\section{Description du modèle}

Les principales caractéristiques et l'architecture fonctionnelle de LX-shore sont illustrées sur la figure 1 et détaillées au travers des sous-sections suivantes.
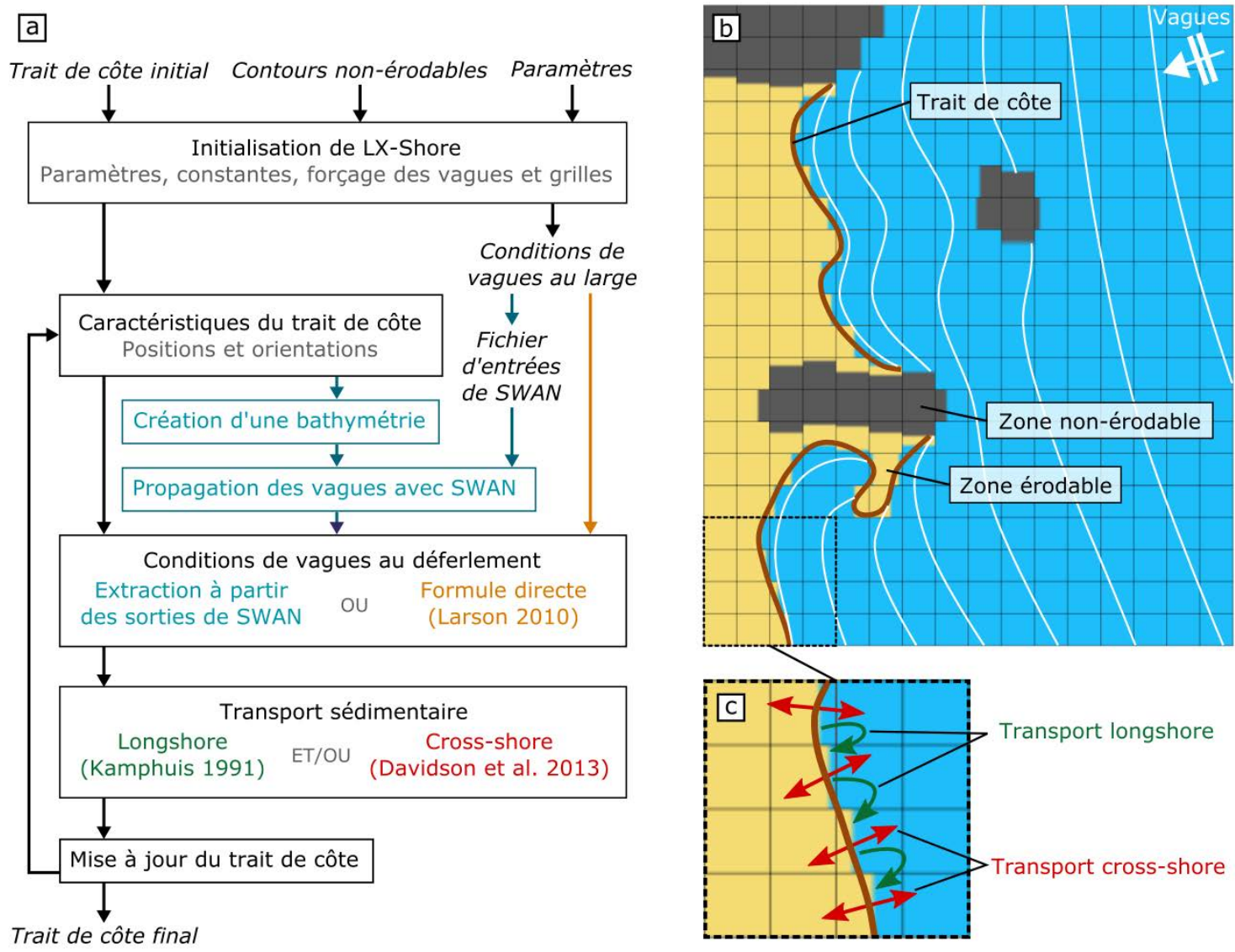

Figure 1. Principales caractéristiques et architecture fonctionnelle de LX-Shore.

Adaptée de ROBINET (2017).

\subsection{Principe}

LX-Shore est un modèle cellulaire 2D en vue de dessus (figure 1b) qui s'inspire du modèle one-line CEM (ASHTON \& MURRAY, 2006) et dans lequel le transport sédimentaire longshore est résolu à l'interface entre les cellules de calcul (figure 1c) par l'application directe d'une formule de transport longshore total intégré à l'échelle du profil de plage actif (jusqu'à la profondeur de fermeture). Il est fait l'hypothèse que tout gradient spatial longshore en transport longshore résulte en un dépôt ou arrachement uniforme et instantané de sédiments le long de ce profil, conduisant à une translation cross-shore du profil et de la position du trait de côte. La formule de KAMPHUIS (1991) est employée par défaut, mais, pour permettre l'application de LX-shore à des cas académiques existants, la formule du CERC (USACE, 1981) peut aussi être utilisée. 


\section{Thème 2 - Dynamique sédimentaire}

LX-Shore intègre une adaptation du modèle ShoreFor (SPLINTER et al., 2014), impliquant un effet mémoire de la plage, qui permet de faire évoluer la position du trait de côte dans les cellules de calcul (figure 1c) en réponse à la variabilité de l'énergie des vagues incidentes des vagues. Tout déséquilibre entre les conditions instantanées et de l'historique proche des conditions de vagues (moyenne pondérée des conditions de vagues vers le passé) génère alors une translation cross-shore de la position du trait de côte. La période de calcul de la moyenne pondérée varie de quelques dizaines de jours pour les plages dont la variabilité du trait de côte est dominée à l'échelle évènementielle (tempête) à plusieurs centaines de jours pour les plages marquées par une plus forte variabilité à l'échelle saisonnière et/ou interannuelle. Dans LX-Shore, le sens de la translation dépend du signe du déséquilibre calculé avec les conditions de vagues au large, tandis que son intensité est proportionnelle au déséquilibre et à la puissance instantanée des vagues au déferlement.

\subsection{Fraction en sédiment et évolution du trait de côte}

La spécificité de ce nouveau modèle qui diffère des approches one-line classiques, est que le modèle ne traite pas directement avec des positions de trait de côte $(x, y)$ mais avec la valeur de fraction en sédiment $(F)$ contenue dans chaque cellule de la grille morphologique qui discrétise le domaine de calcul en vue de dessus (figure $1 \mathrm{~b}, \mathrm{c}$ ) et qui est composée de cellules carrées (maillage structuré) de résolution spatiale de $\sim 100 \mathrm{~m}$. Cette valeur $F$ est interprétée comme la proportion de surface émergée dans chaque cellule $(0 \leq F \leq 1)$. Les cellules d'eau (domaine marin) sont les cellules où $F=0$, les cellules de trait de côte sont les cellules où $F>0$ et ayant un contact de bordure latérale avec les cellules d'eau, et enfin les cellules de terre (domaine terrestre) sont les autres cellules.

A chaque itération du modèle les gradients en transport longshore et le déplacement induit par les processus cross-shore sont calculés pour chaque cellule de trait de côte et sont ensuite exprimés en termes de variation de fraction en sédiment via des relations géométriques simples. Après mise à jour de la valeur de $F$ dans toutes les cellules de trait de côte, des cas particuliers de cellules sur-accrétées $(F>1)$ ou sur-érodées $(F<0)$ peuvent apparaitre. De nombreuses lois de comportement ont été implémentées pour redistribuer les excès et les déficits de fraction en sédiment respectivement vers les cellules d'eau ou de terre adjacentes. Ainsi, la position des cellules de trait de côte dans la grille morphologique évolue au fil de la simulation avec la création et/ou suppression de cellules de trait de côte. L'application d'une méthode de reconstruction d'interface, proche de la méthode PLIC (Picewise Linear Interface Calculation), à la grille de fraction en sédiment permet de retrouver le trait de côte complet à chaque itération et de suivre son évolution au fil de la simulation. Enfin, des zones non-érodables peuvent être définies pour rendre compte de l'existence d'ouvrages de défense ou d'enrochements naturels (figure 1b), élargissant ainsi le champ d'application de LX-Shore. 


\section{XVèmes Journées Nationales Génie Côtier - Génie Civil \\ La Rochelle, 29 au 31 mai 2018}

\subsection{Vagues et bathymétrie}

Comme pour la plupart des modèles de trait de côte à complexité réduite, un modèle de vagues simple (ici formule directe de LARSON et al. (2010) combinée à une méthode d'ombrage linéaire) est utilisé pour obtenir les caractéristiques des vagues au déferlement (figure 1a) avec des temps de calcul très faibles. LX-Shore a aussi été couplé dynamiquement avec le modèle spectral de vagues SWAN (figure 1a,b) pour permettre une application à des côtes complexes où la réfraction et/ou diffraction perturbent fortement les caractéristiques des vagues atteignant la côte. Une grille hydrodynamique ayant une résolution de $\sim 10-20 \mathrm{~m}$ est utilisée pour réaliser les simulations SWAN. Ce choix garantit que la zone de surf couvre dans la direction cross-shore au moins deux à trois cellules hydrodynamiques pour des conditions morphogènes. SWAN propage les vagues au-dessus d'une bathymétrie idéalisée définie sur la grille hydrodynamique et qui est actualisée à chaque nouvelle itération de LXshore selon une méthode similaire à celle utilisée par KAERGAARD \& FREDSOE (2013) mais qui en plus tient compte de la présence de possibles contours de zones nonérodables (ROBINET et al., en révision).

\section{Cas d'application}

\subsection{Processus longshore uniquement}

Tout d'abord deux cas d'application (L1 et L2, figure 2) sont réalisés pour évaluer la capacité du modèle à reproduire des évolutions du trait de côte causées par les processus longshore sur le moyen/long-terme. Seul le transport longshore est utilisé et les conditions de vagues au déferlement sont déterminées avec le modèle de vagues simple. Dans les deux cas d'application le climat de vagues au large est idéalisé par une hauteur significative et une période de vagues constantes $\left(H_{s}=1.5 \mathrm{~m}\right.$ et $\left.T=8 \mathrm{~s}\right)$ et une direction des vagues variable calculée de manière similaire à l'approche utilisée par ASHTON \& MURRAY (2006). Pour chacun des cas d'application, une série temporelle de direction de vagues est générée en sélectionnant aléatoirement des valeurs à partir d'une distribution de probabilité (ROBINET, 2017) définie par son asymétrie (proportion de vagues arrivant par la gauche de la côte) et son obliquité (proportion de vagues ayant un angle d'incidence à la côte supérieur à $45^{\circ}$ ).

Le cas L1 (figure 2a,b) consiste à soumettre une plage initialement rectiligne à un climat de vagues susceptible de développer des instabilités de trait de côte (ondulations, flèches). Il est ici caractérisé par une dominance de vagues provenant de la gauche du domaine et par une forte obliquité à la côte. Le trait de côte est initialement bruité pour exciter les instabilités morphologiques. La simulation est réalisée sur une période de 50 ans avec un pas de temps de 12 heures et une résolution de cellule de $100 \mathrm{~m}$. Dans cette simulation, le trait de côte est instable et des perturbations initiales se développent (figure 2a). Celles-ci s'apparient pour s'auto-organiser dans une longueur d'onde 


\section{Thème 2 - Dynamique sédimentaire}

régulière (régime non-linéaire). L'asymétrie du climat de vagues induit une migration longshore des instabilités et forme des flèches sableuses qui se reconnectent à la côte et cloisonnent des étendues d'eau (figure 2b).

Le cas test L2 (figure $2 \mathrm{~d}, \mathrm{e}$ ) vise à simuler l'impact de la mise en place d'un brise lame au large d'une plage initialement rectiligne et exposée à un climat de vagues symétrique et comportant une faible proportion de vagues obliques. Une période de simulation de 20 ans, un pas de temps de 3 heures, et des cellules de $50 \mathrm{~m}$ sont utilisés. Un salient symétrique se forme progressivement dans la zone d'ombre du brise-lame résultant d'un gradient négatif en transport longshore, et dont l'extension vers large semble saturer à partir de 10 ans $(\sim 80 \mathrm{~m})$, alors qu'un tombolo est formé si le brise-lame est situé plus près de la côte et/ou possède des dimensions longitudinales plus grandes.
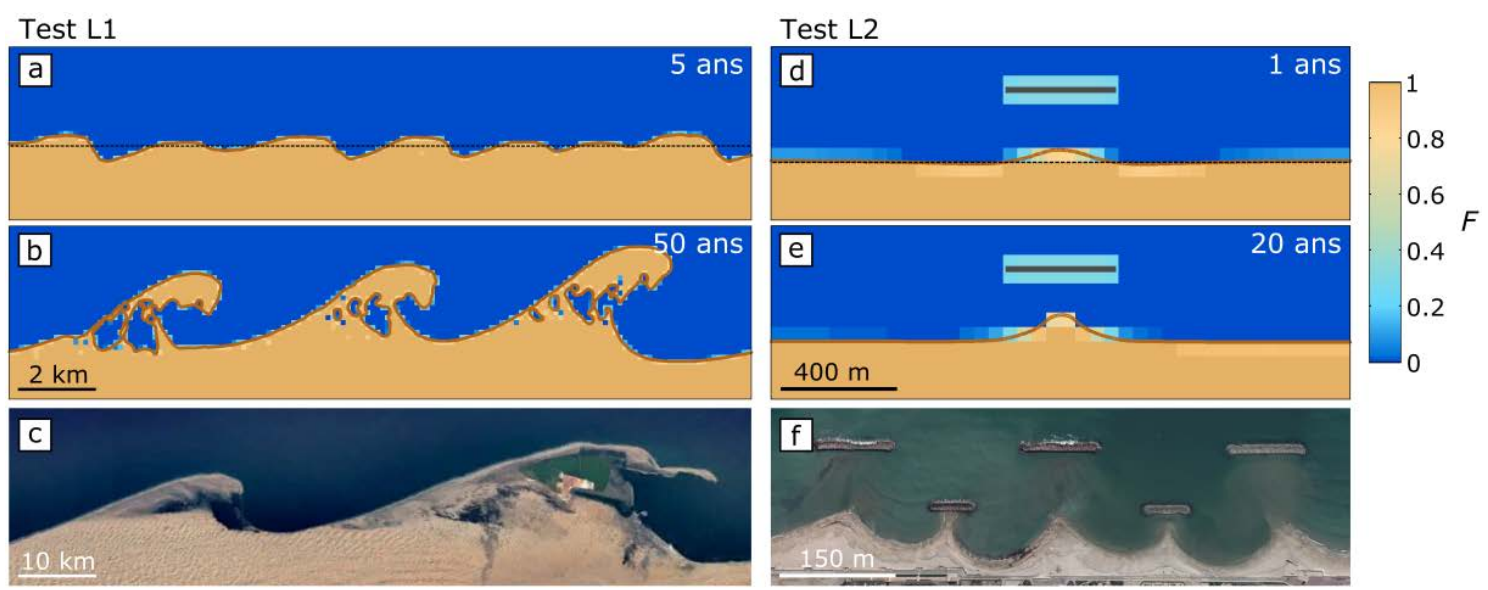

Figure 2. Evolutions simulées pour deux cas académiques (processus longshore uniquement et climats de vagues idéalisés). a,b Développement de flèches sableuses. c Côte Namibienne. d,e Effet de l'installation d'un brise-lame. f Plage de Palavas-lesFlots. Pointillés noirs : trait de côte initial. Adaptée de ROBINET et al. (en révision).

\subsection{Processus cross-shore uniquement}

Un troisième cas d'application (C1) est conduit pour valider l'implémentation numérique et l'adaptation du modèle ShoreFor faite dans LX-Shore. Seul les processus cross-shore sont considérés. LX-Shore est appliqué à la plage du Truc Vert qui est un site pour lequel la contribution des processus longshore dans les évolutions du trait de côte sur le court et moyen-terme est supposée négligeable. Les vagues au déferlement sont déterminées avec le modèle de vagues simple car la côte est rectiligne et les isocontours bathymétriques relativement parallèles à la côte sur le domaine de calcul. La simulation est réalisée sur une période de 9 ans (2005-2014) pour laquelle un jeu de données de validation complet (position du trait de côte et conditions de vagues au large) est disponible. Un pas de temps de 3 heures et une résolution de cellule de $100 \mathrm{~m}$ sont utilisés. Les résultats de simulation (non présentés dans cet article) montrent que 


\section{XVèmes Journées Nationales Génie Côtier - Génie Civil \\ La Rochelle, 29 au 31 mai 2018}

LX-Shore parvient à reproduire une grande part de la variabilité du trait de côte observée sur des échelles allant de l'évènementiel à l'interannuel et avec des performances $\left(\mathrm{r}^{2}=0.67, \mathrm{RMSE}=7.74 \mathrm{~m}\right)$ similaires à celles obtenues dans d'autres études où ShoreFor est directement appliqué à la plage du Truc Vert sur la même période (SPLINTER et al., 2014). Des résultats très similaires sont obtenus lorsque les vagues sont propagées avec SWAN.

\subsection{Plage en baie (processus longshore et cross-shore)}

Un dernier cas d'application (figure 3) est réalisé pour montrer l'apport de LX-Shore dans l'étude de la dynamique du trait de côte sur des sites où les évolutions du trait de côte sont contrôlées à la fois par les processus longshore et par les processus crossshore. Ce cas consiste en une plage initialement rectiligne bordée par deux caps rocheux (figure 3a) et exposée au climat de vagues du cas $\mathrm{C} 1$ sur la même période d'étude.

Un premier test (LC1) est réalisé en utilisant uniquement le transport longshore tandis que le second test inclue également les processus cross-shore (LC2). Les résultats de simulation (figure $3 b, c$ ) suggèrent que dans de telles conditions géologiques et hydrodynamiques, la prise en compte des processus cross-shore ajoute une variabilité dans les évolutions du trait de côte du même ordre de grandeur que celle causée par les processus longshore, sur des périodes et des échelles de temps différentes. De plus, la comparaison des figure $3 \mathrm{~b}$ et $3 \mathrm{c}$ montre que la rotation de la plage induite par les processus longshore domine les évolutions du trait de côte sur la première moitié de la simulation et que la contribution des processus cross-shore prédomine ensuite.

\section{Discussion et conclusions}

LX-Shore incorpore un certain nombre d'approximations physiques qui définissent le champ des applications possibles. Par exemple, il est fait l'hypothèse que le transport sédimentaire cross-shore est uniquement relié à la variabilité temporelle de l'énergie des vagues incidentes et que les contributions respectives des phénomènes de jet de rive et d'overwash sont négligeables. L'impact de la marée est aussi négligé et le plan d'eau est supposé garder une élévation constante durant la simulation. Les courants de marée, estuariens, et induits par les vagues ne sont pas non plus modélisés. Ces hypothèses, inhérentes aux modèles à complexité réduite, sont nécessaires pour maintenir des temps de calcul raisonnables et pour assurer un meilleur contrôle des erreurs numériques pour des simulations sur le moyen et long-terme. Au vu des différentes limitations explicitées dans ROBINET et al. (en révision), LX-Shore vise à être appliqué aux littoraux sableux comportant des dunes/falaises, dominés par l'action des vagues, où les zones rocheuses peuvent être supposées non érodable et sur des échelles de temps allant de quelques heures à plusieurs décennies. 


\section{Thème 2 - Dynamique sédimentaire}
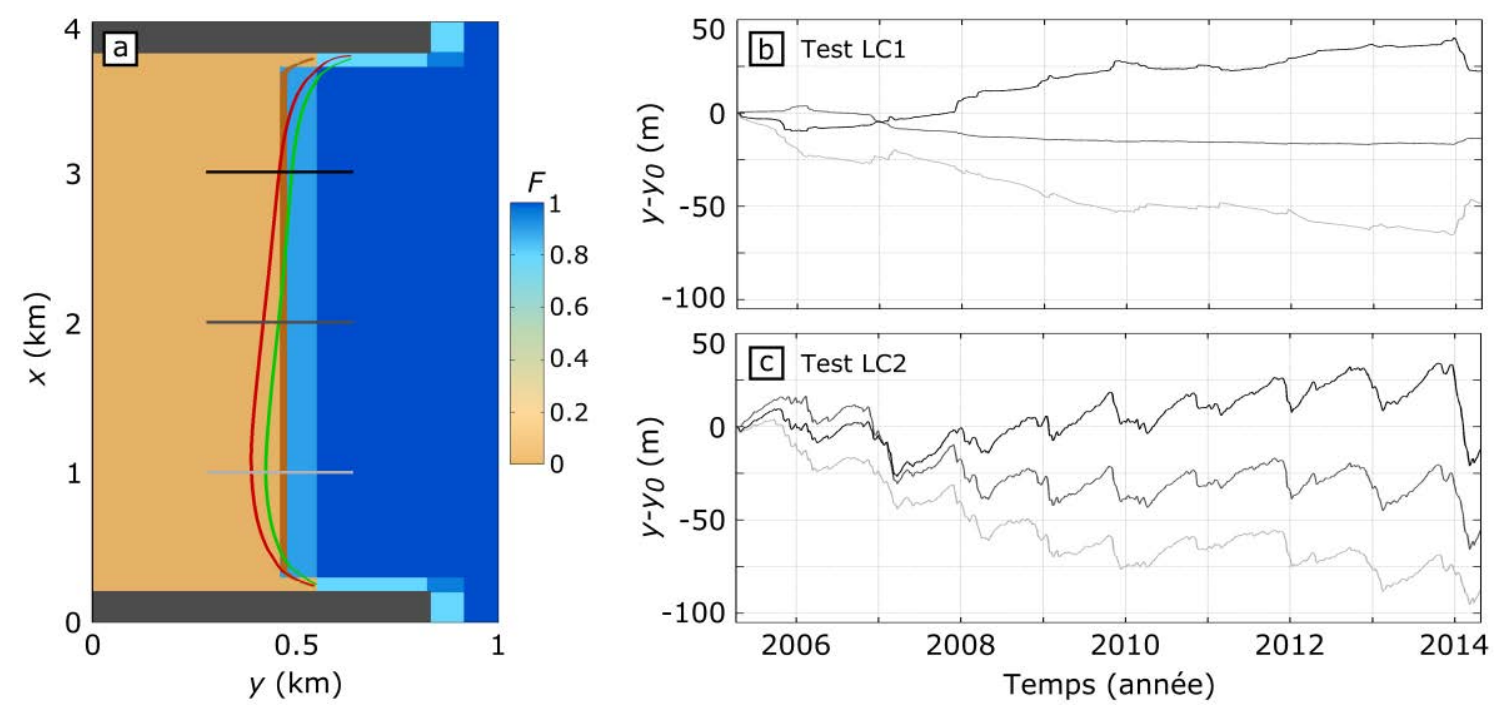

Figure 3. Evolutions d'une plage en baie idéalisée. a Etat initial la plage et du trait de côte (courbe marron). Courbes verte et rouge : traits de côte finaux respectivement associés à (b) et (c). Traits noir, gris et gris clair : transects d'extraction. b Evolution du trait côte aux transects d'extraction avec processus longshore seulement. c Idem que (b) avec processus cross-shore. Adaptée de ROBINET et al. (en révision).

Les cas d'application L1, L2 et LC1 montrent que LX-Shore peut simuler une variété d'évolutions du trait de côte liées à des processus longshore (ex. : instabilités du trait de côte, tendances d'érosion/accrétion résultant de l'implémentation d'ouvrages de défense) sur différentes échelles spatio-temporelles. La comparaison des résultats obtenus pour le cas L1 avec les travaux de ASHTON \& MURRAY (2006) montrent que LX-Shore simule une croissance des flèches sableuses (longueur d'onde et amplitude) plus lente que lorsque CEM est utilisé. Cela s'explique principalement par le fait que CEM incorpore un algorithme d'overwash qui empêche les flèches de se rompre à leur base et qui permet une croissance de ces formes sédimentaires de manière continue vers le large. Des tests additionnels (non présentés dans cet article) révèlent que LX-Shore reproduit le développement d'ondulations de trait de côte (cas où l'algorithme d'overwash n'intervient pas) avec des longueurs d'onde similaires à celles simulées par CEM (ROBINET et al., en révision). La formation d'un salient au droit d'un brise-lame obtenu pour le cas L2 et sa progression vers une forme en tombolo à mesure que l'on augmente le rapport entre la longueur de l'ouvrage et la distance de l'ouvrage à la côte sont des évolutions cohérentes avec les observations et les études portant sur les briselames (ROSATI, 1990). L'application de LX-Shore à des plages où de tels ouvrages ont été installés permettra d'évaluer quantitativement les performances du modèle à reproduire ce type d'évolution du trait de côte. Le cas $\mathrm{C} 1$ confirme que le module crossshore de LX-Shore permet de reproduire fidèlement les évolutions induites par les processus cross-shore de l'évènementiel à l'interannuel. Enfin, le cas LC2 illustre 


\section{XVèmes Journées Nationales Génie Côtier - Génie Civil \\ La Rochelle, 29 au 31 mai 2018}

l'intérêt de disposer d'un tel modèle pour étudier la dynamique du trait de côte pour des sites où les processus cross-shore et longshore coexistent. En effet, par son aspect modulaire et ses nombreuses fonctionnalités, LX-Shore permet de décortiquer la variabilité observée et d'évaluer les contributions respectives des différents processus moteurs.

Les cas d'application présentés ont aussi été réalisés avec différentes résolutions de cellules morphologiques $(-50 \%,-25 \%$ et $25 \%$ de la résolution initiale) pour évaluer la sensibilité du modèle à la taille des mailles. Ces tests additionnels indiquent que le choix de la résolution des cellules impacte peu les évolutions simulées tant que les conditions de stabilité numérique sont respectées (ROBINET et al., en révision). Le faible ratio de temps de calcul sur temps simulé $\left(10^{-4}\right.$ à $10^{-3}$ avec SWAN sinon $\left.10^{-6}\right)$ fait de LX-shore un outil plus adapté à la modélisation long-terme. D'autres développement sont nécessaires pour accroitre le champ des applications possibles du modèle avec par exemple la prise en compte : (1) de sources de sédiments par des rechargements de plages ou des apports de cours d'eau (ex. : ASHTON \& GIOSAN, 2011) ; (2) de la remontée du niveau marin (ex. : BRUUN, 1962); (3) de processus liés à la géologie sous-jacente et d'interactions plage/falaise (ex. : LIMBER \& MURRAY, 2011). Les travaux futurs impliqueront en priorité un travail de validation quantitative en s'appuyant notamment sur le jeu de données de la plage de Narrabeen en Australie (TURNER et al., 2016).

\section{Références bibliographiques}

ASHTON A.D., MURRAY A.B. (2006). High-angle wave instability and emergent shoreline shapes: 1. Modeling of sand waves, flying spits, and capes. Journal of Geophysical Research, Vol. 111 (F4). https://doi.org/10.1029/2005JF000422

ASHTON A.D., GIOSAN L. (2011). Wave-angle control of delta evolution. Geophysical Research Letters, Vol. 38, L13405. https://doi.org/10.1029/2011GL047630

BRUUN P. (1962). Sea-Level Rise as a Cause of Shore Erosion. Journal of the Waterways and Harbors Division, Vol. 88, pp. 117-132.

HARLEY M.D., TURNER I.L., SHORT A.D. (2015). New insights into embayed beach rotation: The importance of wave exposure and cross-shore processes. Journal of Geophysical Research: Earth Surface, Vol. 120 (8), pp 1470-1484. https://doi.org/10.1002/2014JF003390

KAERGAARD K., FREDSOE J. (2013). A numerical shoreline model for shorelines with large curvature. Coastal Engineering, Vol. 74, pp 19-32. https://doi.org/10.1016/j.coastaleng.2012.11.011

KAMPHUIS J.W. (1991). Alongshore Sediment Transport Rate. Journal of Waterway, Port, Coastal, and Ocean Engineering, Vol. 117 (6), pp 624-640, https://doi.org/10.1061/(ASCE)0733-950X(1991)117:6(624) 


\section{Thème 2 - Dynamique sédimentaire}

LARSON M., HOAN L.X., HANSON H. (2010). Direct Formula to Compute Wave Height and Angle at Incipient Breaking. Journal of Waterway, Port, Coastal, and Ocean Engineering, Vol. 136 (2), pp 119-122. https://doi.org/10.1061/(ASCE)WW.1943-5460.0000030 LESSER G., ROELVINK J., VAN KESTER J., STELLING G. (2004). Development and validation of a three-dimensional morphological model. Coastal Engineering, Vol. 51 (8-9), pp. 883-915. https://doi.org/10.1016/i.coastaleng.2004.07.014

LIMBER P.W., MURRAY A.B. (2011). Beach and sea-cliff dynamics as a driver of long-term rocky coastline evolution and stability. Geology Vol. 39, pp. 1147-1150. https://doi.org/10.1130/G32315.1

ROBINET A., CASTELlE B., IDIER D., LE COZANNET G., DEQUE M., CHARLES E. (2016). Statistical modeling of interannual shoreline change driven by North Atlantic climate variability spanning 2000-2014 in the Bay of Biscay. GeoMarine Letters, Vol. 36(6), pp 479-490. https://doi.org/10.1007/s00367-016-0460-8

ROBINET A. (2017). Modélisation de l'évolution long-terme du trait de côte le long des littoraux sableux dominés par l'action des vagues. Thèse Université de Bordeaux, Bordeaux. Disponible en ligne sur URL : https://tel.archives-ouvertes.fr/tel-01755070

ROBINET A., IDIER D., CASTELLE B., MARIEU V. (en révision). A reducedcomplexity shoreline change model combining longshore and cross-shore processes: the LX-Shore model. Environmental Modelling \& Software

ROELVINK D., RENIERS A., VAN DONGEREN A., VAN THIEL DE VRIES J., MCCALL R., LESCINSKI J. (2009). Modelling storm impacts on beaches, dunes and barrier islands. Coastal Engineering, Vol. 56 (11-12), pp. 1133-1152. https://doi.org/10.1016/j.coastaleng.2009.08.006

ROSATI J.D. (1990). Functional design of breakwaters for shore protection: empirical methods. Technical Report, US Army Corps of Engineers, Coastal Engineering Research Center, CERC-90-15

SPLINTER K.D., TURNER I.L., DAVIDSON M.A., BARNARD P., CASTELLE B., OLTMAN-SHAY J. (2014). A generalized equilibrium model for predicting daily to interannual shoreline response. Journal of Geophysical Research: Earth Surface, Vol. 119 (9), pp. 1936-1958. https://doi.org/10.1002/2014JF003106

TURNER I.L., HARLEY M.D., SHORT A.D., SIMMONS J.A., BRACS M.A., PHILLIPS M.S., SPLINTER K.D. (2016). A multi-decade dataset of monthly beach profile surveys and inshore wave forcing at Narrabeen, Australia. Scientific Data, Vol. 3, 160024. https://doi.org/10.1038/sdata.2016.24

USACE (1984). Shore protection manual. Technical Report, US Army Corps of Engineers, Coastal Engineering Research Center. https://doi.org/10.5962/bhl.title.47829

VITOUSEK S., BARNARD P.L., LIMBER P., ERIKSON L., COLE B. (2017). A model integrating longshore and cross-shore processes for predicting long-term shoreline response to climate change. Journal of Geophysical Research: Earth Surface, Vol. 122(4), pp 782-806. https://doi.org/10.1002/2016JF004065 